\title{
La degradación de un sistema económico que no atisbó la educación en consumo sostenible. Crítica social.
}

\author{
Ma Aurora LóPEz LÓPEZ \\ Universidad de Valladolid \\ maria.aurora22@gmail.com \\ Luis RoDrigo MARTÍN \\ Universidad de Valladolid \\ lrodrigo@hmca.uva.es \\ Isabel RODRIGo MARTíN \\ Universidad de Valladolid \\ Isabel.rodrigo@uva.es
}

\section{Resumen}

Desde un enfoque multidisciplinar y asentado sobre la crítica social, el presente artículo estudia nuestra realidad económica, de consumo, y también educativa, poniendo de manifiesto incoherencias en la gestión de nuestro sistema, por parte de nuestros gobiernos y a nivel individual, describiendo un "modus operandi" rutinario que soporta estilos de vida inadmisibles en tiempos de crisis. La insostenibilidad de esta forma de proceder y gestionar la realidad económica y social, atisba también las carencias en formación de la ciudadanía, debido a un sistema educativo obsoleto. Sólo la educación en consumo podrá transformar la realidad y nuestro futuro devenir.

Palabras clave: economía, educación, consumo, sostenibilidad.

\section{A degrading economic system was not understood to educate on sustainable consumption is the key to future. Social criticism.}

\begin{abstract}
From a multidisciplinary perspective, is based on social criticism, this article examines our economic, consumer, and education, in which it's obvious what inconsistent is our management system, not only for the activity of our governments, but also individually. It allows describing a "modus operandi" that frequently draws inadmissible lifestyles to support in hard times. The invalidity of this procedure and way of managing the economic and social reality, also catchs a glimpse the lack of training in citizenship, due to an obsolete education system. Only consumer education will transform our reality and bring us a sustainable future.
\end{abstract}

Keywords: economy, education, consumption, sustainability.

\section{Referencia normalizada:}

López López, M. A.; Rodrigo Martín, L. y Rodrigo Martín, I. (2013) La degradación de un sistema económico que no atisbó la educación en consumo sostenible. Crítica social. Historia y Comunicación Social. Vol. 18. $\mathrm{N}^{\circ}$ Especial Diciembre. Págs. 115-127. 
Sumario: 1. Introducción. 2. Metodología. 2.1. Retroceder económicamente para avanzar. 2.2. Trueque para el consumo. 2.3. Educar innovando. 3. La disfuncionalidad del sistema actual. 4. Conclusiones. 5. Referencias bibliográficas.

\section{Introducción.}

La presente investigación parte de la observación de una serie de carencias vinculadas a nuestro sistema de gobierno, y de vida en general, en el que se aprecian graves disfunciones en lo referente a la interrelación más coherente que debería de existir entre los sistemas económicos, los sistemas de consumo, y los de tipo educativo.

La premisa, por tanto, que definiría esta idea estaría configurada en torno a un pensamiento que, debido al momento actual, cada vez tiene un peso mayor, si se analiza rigurosamente el sentimiento colectivo de la población, quejumbrosa, con bastante frecuencia, de tanta incoherencia como registra a su alrededor.

A diario, la dificultad inherente ante cualquier acto y o movimiento que tenga lugar es fácilmente indiscutible; los inconvenientes que cualquier gestión acarrea al ciudadano, afloran con rapidez, se trate de lo que se trate; y la lógica y el sentido común aplicable a cualquier asunto cotidiano, se pierde entre las jerarquías de un sistema completamente obsoleto y desbordado por falta de modernidad, adaptación y auto-reflexión.

Las exigencias de cambio siempre recaen en el ciudadano, mientras el sistema se presume avanzado y eficiente, cuando no alardeé también, de una eficacia en numerosas ocasiones completamente irreal.

Dicho esto, y si prestamos atención a tres materias vitales, que en apariencia distintas, se encuentran estrechamente relacionadas, como son: la economía, el consumo y la educación, podremos comprobar cómo nuestras primeras líneas van cobrando sentido. Tan sólo necesitamos presentar con la suficiente vehemencia evidencias certificadas susceptibles de consagrarse en una declaración muy crítica de nuestra realidad, dispuesta a ser escuchada por una minoría todavía muy dimunita (desafortunadamente) para que todo ello pueda cambiarse y mejorar.

Sin embargo, aparte de lo interesante que pueda resultar abordar un fenómeno de descoordinación certera entre los sistemas económicos, en los encontramos indiscutiblemente a su vez implícitos los de consumo, y ello vaya unido también al "modus operandi" del sistema educativo, nuestro estudio cobrará razón especial, y muy significativa, por aproximarse a todo este conocimiento desde una perspectiva en todo momento consciente de la importancia de la globalización, y del modo en el que ésta planea sobre todas las cuestiones vitales, en su afán de reestructurar con determinismo y justicia global la integridad y sostenibilidad que ya obligatoriamente se dice que ha marcar nuestro rumbo futuro. 
¿Estaremos ante el camino que nos lleva a conseguirlo? ¿Habremos alcanzado ya el grado de modernización necesario para asumir los fuertes cambios que nos esperan? ¿Ha cambiado lo suficiente la mentalidad de nuestros pueblos, como para transformarse realmente en esa suma ingente de intenciones a diario vertidas en forma de palabras por cualquier ser humano con independencia de su estado, trabajo, profesión,...?

\section{Metodología.}

Este trabajo se fundamenta principalmente sobre la base de una creciente aparición de programas televisivos, noticiarios, debates públicos y/o privados, reportajes, crónicas, libros, artículos, y cualesquiera otras fuentes de orden secundario, que destacan por lo ambivalente de sus temáticas, con frecuencia dirigidas hacia un análisis constante y permanente de la situación de crisis política, social, económica, administrativa, educativa, etc., que estamos atravesando en nuestro país, y cuya proyección, de manera generalizada, también se deja notar, si centramos nuestra atención sobre otras zonas geográficas del mundo.

Habiéndose escogido un enfoque claramente orientado hacia la crítica social, como la perspectiva de estudio desde la que moldear y asumir la interrelación de los contenidos investigados, que indican tanto pesimismo en el futuro, la falta de confianza en nuestros sistemas y el descontento popular que se desprende de las declaraciones cotidianas de los españoles tanto en las encuestas informales como en las formales, ya profesionalmente concebidas, más una suma muy relevante de conocimiento específico directo sobre las materias vinculantes: economía, consumo y educación, es como se lleva a cabo este ensayo, que pretende ofrecer un resultado en el que la protagonista se llame: reflexión.

Las contribuciones multidisciplinares se erigen responsables de la excelencia introspectiva inmersa en este documento, elaborado gracias a esas otras aportaciones más extensas que conforman el proyecto de procedencia y referencia, mucho más ambicioso y de mayor envergadura consecuentemente.

Dado, que sin duda alguna, el nuevo empoderamiento social se denomina naturalmente "capacidad de cambio", es que nos hemos confinado en esa línea de determinación capaz de descifrar dónde están las claves del mismo y qué necesitamos para poderlo conseguir, destapando asimismo nuevas vías de desarrollo y convocando a una permutación necesaria de nuestra mentalidad actual.

Como temática clave implícita se acude a la globalización, en tanto en cuanto, su comprensión se sitúa hoy en día como la idea desde la que proceder ante cualquier reestructuración del sistema, ya sea política, social, económica, educativa, científica, filosófica, y en definitiva, existencial. 
Visto así, los objetivos principales que argumentan este escrito y facilitan la identificación de los parámetros capaces de clasificarlo, se pueden concretar sutilmente en los siguientes:

- Analizar desde un enfoque socialmente crítico y a grandes rasgos la situación de crisis actual.

- Analizar desde un enfoque socialmente crítico nuestro actual sistema económico.

- Analizar desde un enfoque socialmente crítico nuestro actual sistema de consumo.

- Analizar desde un enfoque socialmente crítico nuestro actual sistema educativo.

- Interrelacionar los resultados recogidos de los análisis críticos para la visualización de un nuevo escenario de cambio necesario.

- Valorar y entablar nuevas vías de desarrollo factibles de practicarse y considerarse, de cara a la materialización de ese nuevo escenario de cambio planteado con urgencia.

- Aportar una nueva forma de comprender nuestra realidad social, económica y educativa, que consiga esclarecer cuál sería la gestión apropiada y apta para el funcionamiento coherente y coordinado de los tres sistemas objeto de nuestro estudio.

De este modo, se entiende, que el soporte teórico que sustenta este estudio, converge en una atención pluridisciplinar sobre diferentes ciencias y disciplinas, entre las que cabe destacar: la economía, la sociología, las ciencias políticas, la educación, la filosofía, la psicología, el consumo y el marketing, entre las más importantes.

Un último dato a nivel metodológico y muy interesante, es la finalidad retrospectiva que metafísicamente conlleva la narrativa de cada alegato suscrito.

\subsection{Retroceder económicamente para avanzar}

Uno de los aspectos fundamentales que cursan la trayectoria de nuestra investigación responde a la implorante necesidad de retroceso económico al que una afluencia desmesurada de variopintos mensajes han constituido en el presente reclamo social.

Rebajar nuestro nivel económico de vida está hoy de moda, y lo que es peor, es condición obligada para todo viandante, o dicho de otro modo, para los que transitamos con rutina las calles de nuestra nación, para los que estamos expuestos al quehacer diario social: encontrarnos a la vecina, topar con el jefe despiadado y prepotente, realizar cincuenta mil trámites administrativos hasta conseguir cambiar nuestra dirección fiscal de notificaciones, llamar cien veces por teléfono a este o aquel sitio hasta conseguir que te atiendan, comprar el pan y conducir tu propio coche tanto para cortas como medianas y grandes distancias. Hablar del resto de personas ajenas a este círculo estaría demás ahora, además ¿para qué? Seguro que ellos ya saben cómo interpretarlo, y algo mucho mejor, cómo gestionarlo, de cara a su continuidad o no. 
Por tanto, que nuestra capacidad adquisitiva sea hoy menor que nunca es un hecho no sólo evidente, sino que se nos ha impuesto. Pero, quizá, lo más preocupante sea, no saber hasta cuándo tal circunstancia perdure. Sin embargo, esta larga racha, puede acortarse y superarse. La clave es sencilla, emprenderla no tanto, y comprenderla el punto de partida que nos lleve a satisfacer la nueva necesidad de cambio. La satisfacción de las necesidades es una cuestión vital, pero el modo en que se haga no es invariable, y es aquí donde hemos pecado de acomodación, y donde nos hemos rendido ante un sistema inhumano. El pago de cualquier intercambio no tiene únicamente porqué resolverse mediante un traspaso monetario.

Esta es la idea que representa la frustración que define a todos aquellos ahora limitados económicamente, más cuando sus vidas tienen lugar en estados fuertemente desarrollados, y en los que el dinero se presenta como único recurso para hacer efectiva la transacción de un bien o servicio.

Si miramos atrás, con agilidad se presenta ante nosotros la imagen de que cualquier tiempo pasado fue mejor, y con poca presteza recordamos que a cualquier avance de la humanidad le antecede una época de escasez, de caos, de tragedia, de fracasos, olvidando a su vez que sirvieron al progreso.

La idea de progresar en la vida se ha ligado en los últimos años a algo tan trivial como el consumo banalizado, es decir, el consumo desarraigado de necesidades reales, que con el paso del tiempo, se iban tornando más caprichosas y descomedidas que aceptables, y lo que resulta más crítico aún, soportado sobre la base de un crédito ficticio de capital líquido. Y los problemas derivados del dinero a crédito, así como de la desigualdad de acceso al mismo, ya nos los explica Debraj Ray (2002).

“¿Dónde está el dinero?” Se pregunta la gente. "Se lo han llevado”, dicen. Ciertamente son muchos los casos de corrupción, muchos los fraudulentos, pero la mayoría de todo lo que ha salido de este país, no existía; no era real. Ese ha sido uno de los grandes problemas, que desde el más pudiente hasta el más pobre estaba endeudado por algún tipo de alocada inversión.

Cuando algo se presta y no se devuelve, se provoca con ello tensión, se despierta en el prestamista su sentido más hábil de la estrategia, esa que finalmente le permita recuperar aquello que dejó. Parece fácil así contado, pero cuando los dólares y los euros se pasean entre las manos, todo se vuelve excesivamente complejo.

Sea como fuere, ahora debemos retroceder, por imposición y por obligación, y porque la codicia de unos, la ignorancia de otros, y la falta de sentido común entre todos, se ha topado de frente con toda la población, sometiéndola a un nuevo ritmo, que ahora ya se cierne desagradable, y eso en la masa, acaecido en la multitud, no puede sino traer la revelación ante el sistema y la implantación de un nuevo rumbo.

Si retroceder económicamente es un hecho ya inevitable, no malgastemos energías en verlo negativo, pues aún con mucha pesadumbre, y para vencer la adversidad, será sin duda más inteligente reflexionar y visionar la realidad con positivismo para dar 
con la salida, para avanzar. De lo contrario nuestro sometimiento e ineptitud ante la caída del paraíso crecerá.

\subsection{Trueque para el consumo}

No es por rememorar tiempos antiguos, lejos de nuestro presente, pero el trueque está llamando a la puerta de las economías imposibilitadas de cualquier ciudadano. La pena es que no le abrimos la puerta, seguimos obstinados en esperar a que todo esto pase y de nuevo vuelva el momento oportuno para el despilfarro, la prodigalidad, la abundancia.

Nada como no aprender de los errores puede traernos más desgracias, si cada uno de nosotros no asume su parte del todo, no asumirá tampoco su responsabilidad, y en el menor de los despistes se verá nuevamente sorprendiendo, atravesando la misma situación. Hay que ser prácticos, y cuán más inmediato esto sea mejor. Vivimos muy deprisa, y cualquier asunto que no trate con realismo todo aquello relativo a nuestro día a día nos resulta de poco interés, no es digno de nuestra atención.

Esta incapacidad de reflexión nos impide para ser más eficientes, nos vulnera y nos maltrae por un camino de desolación, donde no hay apenas sabor a gloria, y en el que los pensamientos negativos se vuelven obsesivos y circundantes hacia una sola temática: querer consumir, y morir en el intento. El consumo ha sobrepasado la naturaleza de nuestra especie, se ha inmiscuido dentro de nuestro código genético, y nos creemos infelices cuando no lo podemos satisfacer.

Pero la mayoría de estudios sobre consumo versan sobre las emociones, sobre el deseo compulsivo de adquirir productos y contratar servicios, porque dicho ejercicio va acompañado de un consumo de sensaciones tan socialmente admitidas y reconocidas que forman parte de nuestra identidad.

Las marcas constituyen en los mercados los símbolos desde los que dignificar lo humano, como si realmente existieran categorías superiores e inferiores de nuestra especie. Parece de locos, pero es algo normal, el consumo social es irreversible, y esto es sumamente trascendente. Esta manifestación nos lleva a entender que no es cuestión de acabar con el mismo, es cuestión, de hacerlo sostenible, porque esta última crisis, principalmente financiera, ha venido a demostrar que la mayor dificultad reside en que el capitalismo ha convertido al consumo social en insostenible, porque se ha autoexprimido tanto, que se ha vencido así mismo, ha quebrado, y ello no quiere reconocerse.

Se ha bajado la calidad, en detrimento de la cantidad, y para darle solución, se ha visto más oportuno menguar las opciones de diversidad de consumo para la mayoría, y ensalzar y agrandar las de una minoría rica, precisamente porque los intereses económicos de esos que se enriquecen a costa de la pobreza de otros no cesan. Por señalar culpables, miremos hacia arriba, esto sucede también en aquellas zonas potencialmente más fuertes, con más recursos, como por ejemplo Estados Unidos, que es la primera potencia mundial (Chomsky y Ramonet, 2008: 30). 
Puesto que el consumo de masas, es un acto más afectivo que económico, debemos valorar con seriedad la posibilidad de intercambio que esto nos ofrece. La confianza que hace efectiva en los negocios el dinero para que fructifiquen, puede sustituirse por la palabra de las personas. En el fondo, no es lo uno más que lo otro, el riego es el mismo; cuando hay un cambio material que saldar con dinero, la pérdida puede ser la misma, que cuando en el negocio se busca intercambiar algo de tu propiedad, por otra cosa o servicio de un tercero, debido a unos intereses compartidos que en un principio ambos desean satisfacer. Ha de haber acuerdo, pero eso es todo lo que se necesita.

De este modo, por una parte, se conseguiría suplir la capacidad de intercambio económico por ésta otra, sujeta simplemente al ejercicio del trueque. Se conseguiría con ello, ampliar las opciones de consumo de una gran parte de la población que actualmente está atravesando dificultades económicas fuertes, y que impedirán por este motivo también a sus generaciones descendientes el alcanzar con solvencia la calidad de vida deseada, proclamada y esperada por una sociedad que se prevé en continuo progreso, gracias a la modernización de sus vidas mediante una supuesta evolución tanto a nivel económico, como político, filosófico, educativo, sanitario, y social. El sistema sería más justo, y las diferencias más asequibles, puesto que desde un punto de vista pragmático, en la toma de decisiones final tendría un peso mayor y más real la emoción, los sentimientos, de manera que el consumo se humanizaría más, el sentido de lo económico sería más relativo para todo, y ello repercutiría en una mayor sostenibilidad del propio sistema de vida, más equitativo, en base a oportunidades de desarrollo menos mercantilistas, en cuanto al significado puramente monetarista de la palabra se refiere.

\subsection{Educar innovando}

El concepto de innovación en educación es el siguiente y último que nos incumbe para comprender a la postre la disfuncionalidad de nuestro sistema actual. En primer lugar, hemos de decir que la educación es un tema delicado; genera controversias, porque al igual que todo, siempre es susceptible de mejorarse, pero todavía más efervescentes y sonadas, porque al igual que como sería en el caso de la sanidad (de la que depende la calidad de vida de toda la población), de ella depende la calidad moral y profesional de las personas que conforman nuestra sociedad, con independencia de su lengua, ideología política y religiosa, liquidez, etc.

La peor lacra que puede sobrevenirle a la educación es que ésta se politice, y precisamente esto es algo ya demasiado recurrente, que además de ser un hecho constatado, pues la legislación referente a educación cambia en nuestro país al compás de los gobiernos, aún no se atisban las fórmulas que impidan que haya mayor consenso y esto deje suceder.

Tanta politización, complica además la efectividad de la misma, y que la educación se trate en consideración hacia aquello que realmente es conveniente considerar con respecto a la misma, y cuya atención se desmerece gracias a esa guerra ideológica que 
mantiene a nuestros gobernantes permanentemente enfrascados en cuestiones que finalmente son de orden político y no educativo.

Cuando, señalamos la importancia de educar innovando, forma parte de nuestros objetivos el aclarar que desde nuestro punto de vista, la educación se ha quedado obsoleta conforme a los tiempos que corren. Los diferentes cambios de vida de los últimos años, y el estancamiento que la sociedad está experimentando en numerosos terrenos y ámbitos, tampoco ha pasado de largo para la educación, ya que su actividad en colegios e institutos se mantiene a imagen y semejanza de cómo se lleva ya haciendo durante los últimos 30 años, prácticamente desde que tuviera comienzo la transición.

Con independencia de la ley regente, puede decirse que en la escuela, ya sea en primaria y/o secundaria, el alumnado está sometido al estudio de ciertas materias cuyos contenidos apenas varían, por lo que con carestía se renuevan. Matemáticas, lengua y literatura, historia, filosofía, inglés, física, biología, etc., Entre todas ellas ha resultado bastante discutida la impartición de la Religión Católica Apostólica, cuya nota todavía computa a la hora de establecer la media final definitiva de los expedientes de los alumnos de bachillerato, tan considerada de cara a los procesos de selectividad que permiten escoger una carrera y entrar en la universidad, o el acceso a la formación profesional.

Es por esto, y por la especialización que nos compete, lo sorprendente que resulta comprobar lo desatendido que tiene el sistema educativo el mundo del consumo, que el estudio del mismo le pasa desapercibido, y que sin reparar en ello, como si no fuera importante, continúa su actividad ajena al mismo; y ya la afirmaba Pujol i Villalonga (1996) cuando señalaba la importancia que tenía formar desde la escuela al consumidor. ¿Cómo puede consentirse que en nuestros colegios e institutos no exista educación para el consumo cuando la sociedad hoy en día no puede explicarse sin atenderse a este fenómeno?

Educar innovando, no es una idea de apego a la incorporación de avances tecnológicos, mediante los cuales aumentar el patrimonio material de nuestro alumnado, de nuestros futuros seres sociales, porque en eso nos convierte la educación, en seres sociales, que no en animales, que es lo que ya somos. Innovar en educación es incorporar los avances de la humanidad a su sistema de aprendizaje, para que éste los muestre tal como son y cómo podrían ser, para ampliar puntos de vista desde los que comprender la inteligibilidad social, para que el futuro esté en manos de aquellos capaces de gestionarlo; el futuro en manos del futuro, no un futuro en manos del pasado o, de cómo mucho, de un presente estanco.

Asusta ver cómo ha evolucionado el comercio en las sociedades avanzadas, como su despliegue ha vuelto complejo numerosos sectores antes excesivamente sencillos; cómo la industrialización y la mejora tecnológica han acabado por confundir función con funcionalidad, rendimiento con rendición, calidad con cantidad, y así infinidad de errores. Y no siendo todo un error, y contándose también a pares los logros conseguidos, es ahora el momento, por su carácter inéditamente crítico, de autoexigirnos, 
y reivindicar que el sistema incorpore aquello que ha adquirido un carácter vital, y el consumo y su sistema de funcionamiento, que responde a la infraestructura logística, así como a la configuración de los sistemas de producción que se levantan en pro del mismo, son cuestiones cruciales en las que todo individuo se debe formar. Para responder con criterio antes los cambios económicos, sociales, políticos, etc., primero debemos adoptar un conocimiento profundo sobre el consumo, porque su acto se ha trivializado tanto en este siglo, -que pasará a la historia como aquel por excelencia de la comunicación interpersonal, gracias a la destrucción de las barreras físicas que disminuían la capacidad de relación bidireccional de unos sectores demográficos con otros-, que todo es consumible, todo tiene un valor de intercambio y todo requiere de un acuerdo de transacción. Si conseguimos analizar las consecuencias que ello tendrá, y trasladarlas a la población desde un ejercicio educativo coherente, estaremos muy cerca de alcanzar que las riendas de nuestras vidas sean más fácilmente dirigibles por nosotros mismos y dependen menos de aquellos que malinterpretan el sentido oficial de la globalización (Varis, 2010), dándole ese que se merece: favorecer una riqueza multicultural conjunta, procedente de una suma de partes cuya convivencia se rija por el respeto mutuo, y la primacía de la diversidad frente a la homogeneidad, gracias a una idónea y modernizada educación en materias y valores.

\section{La disfuncionalidad del sistema actual}

La incongruencia patente en la vigente constitución de nuestro sistema económico, desde el que se pretende impulsar un gasto en consumo, imposibilitado por las acciones previas que determinan el bienestar del otro, del sector financiero, que crea oportunidad de ingresos, de trabajo, de inversión, merece un estudio detallado, que pueda asumir con el realismo pertinente, el establecimiento de medidas que sirvan en la generación de una prosperidad en el medio-largo plazo.

La libre competencia, y el aperturismo de las fronteras para osadía de ésta en pro del libre mercado, ha superado los fines que la crearon, y de manera ilógica y sin consciencia firme sobre ello, se ha ido dejando el comercio en la mano de los comerciantes, el dinero en las de los banqueros, las leyes en las de los políticos, ¿y el orden? ¿La vida? También en las mismas manos. Los sectores productores no han encontrado otra vía de escape para no perecer y continuar subsistiendo que la de rendirse ante sus determinaciones (ver informe de la OIT, 2002); las personas, toda la sociedad entera, se ha visto como nunca antes sacrificada ante el pago inapropiado que ha tenido que afrontar de una deuda impune resultante de malas gestiones, por la falta de profesionalidad, por un lado, y del sentido del deber y de la responsabilidad por otros, y en definitiva, por una gran falta de educación en las personas que llegan a ocupar puestos de mandato y relevancia de tal envergadura, de tanta como para disponer cuál será el porvenir de generaciones enteras. 
Considerando esto, podríamos comenzar a subsanarlo con más y mejor educación. Pero si no incorporamos a nuestro sistema educativo los avances y los logros de la impronta de nuestra evolución, y priorizamos únicamente aquello relativo a la economía y su cuestionable forma "sana" de concebirse aún todavía hoy en día, estaremos ante un fracaso que no tendrá cura ni en 20, ni en 50 años, sino pasados muchos siglos más.

Un ejemplo que evidencia dicha carencia en la práctica real es el siguiente. Antiguamente, las personas compraban en tiendas pequeñas, normalmente especializadas, y se autoabastecían también, especialmente en las zonas rurales, de alimentos que ellos mismos criaban. Ahora hay niños en nuestras ciudades que jamás han visto un plantación de hortalizas, no saben si la almendra crece en un árbol, ni que aspecto tiene su fruto, ni como es una platanera, ni en qué época de forma natural se pueden producir qué alimentos, ni qué precio tienen en los mercados, desde su origen hasta su obtención definitiva para el consumo, y esto es inaceptable pues el niño es una "esponja" que absorbe conocimientos sin parar, que realiza asociaciones, y que ello le permite relacionarse con el medio que le rodea (Rollano Vilaboa, 2004: 2) ¿Entonces? Y no solamente los niños, los adultos también, porque muchos desde que tienen uso de razón ya han visto a sus padres consumir en supermercados, comprar el pan prefabricado y congelado para hacerlo en el horno de casa, y no saben ni distinguir qué zona de cada parte de un animal sirve a un tipo de cocinado diferente, y así comerlas adecuadamente, e interminablemente podríamos continuar aludiendo de este modo a todo lo que se consume en la actualidad. La ignorancia en este sentido es total, y como cabría también decirse, atrevida, porque mediante nuestras decisiones de compra, estamos colaborando en la construcción de una cadena de producción y consumo, que se aprovecha precisamente de ese analfabetismo consumista, de esa falta de conocimiento, que como se está comprobando está invitando a la creación de un sistema de abastecimiento de corte imperialista, afanado en la explotación de los recursos y en degradar las condiciones de salubridad de las personas.

\section{Conclusiones}

Consumir es necesario, y la dignificación social del consumo lo es también, pero ello no ha de pasar por constreñir su sentido conforme a un lucro insolente y desvergonzado, por parte de aquellos que se benefician de ponerlo a nuestro alcance. Y esto no es una impresión, prueba de ello es el sentimiento creciente en la población en contra de la gestión del consumo que realizan las grandes empresas de la distribución, que menguan la capacidad de negociación y comercialización que pueden llevar a cabo los fabricantes y productores de sus bienes y cosechas. La pleitesía y fervor de la gente no está ya al nivel de sus comienzos, porque la excesiva proliferación de sus formatos, ha contribuido también a un ejercicio de la competencia entre firmas, cuya desfachatez tampoco ha pasado ante el consumidor inadvertida. 
Aunque de manera prematura y un tanto débil, la sociedad y sus modos de consumo están cambiando, y una nueva buena asoma con optimismo de cara al futuro, solicitando mesura, sostenibilidad, y una gestión menos egoísta de los intereses interpuestos en el camino, desde el origen hasta el final de los pasos que hacen posible el acto de consumir.

Por lo demás, para decir aún una palabra sobre su pretensión de enseñar cómo debe ser el mundo, la filosofía llega siempre demasiado tarde. Como pensamiento del mundo sólo aparece en el tiempo después en el que la realidad ha cumplido su proceso de formación y ha terminado. Lo que enseña el concepto lo muestra necesariamente igual la historia, de modo que sólo en la madurez de la realidad aparece lo ideal frente a lo real y se hace cargo de este mundo mismo en su sustancia, erigido en la figura de un reino intelectual. Cuando la filosofía pinta su gris sobre gris, no se deja rejuvenecer, sino sólo conocer; la lechuza de Minerva sólo levanta su vuelo al romper el crepúsculo. (Hegel, 2005: 21).

\section{Referencias bibliográficas.}

BAUDRILLARD, J. (2009).La sociedad del consumo. Sus mitos, sus estructuras. Madrid. Siglo XXI de España Editores.

BELL, D. (1996). The Cultural Contradictions Of Capitalism: 20th Anniversary Edition. Basic Books. New York.

BOURDIEU, P. (1999). La miseria del mundo. Madrid. Ediciones Akal.

CASTELLS, M. (1999). La era de la información. Economía, sociedad y cultura. Madrid. Alianza Editorial.

DE JUAN LÓPEZ, S. (2009). Thomas Hobbes o la unidad de naturaleza y sociedad. Barcelona. Ediciones Erasmus.

DEBRAJ, R. (2002). Economía del desarrollo. Barcelona. Antoni Bosch Editor.

DELORS, J. (1996) La educación encierra un tesoro. Madrid. UNESCO-Anaya.

FAURE, E. (1973). Aprender a Ser. París. UNESCO.

FERMOSO, P. (1994). Pedagogía social. Fundamentación científica. Barcelona. Herder.

HAKIM, C. (2012). El capital erótico: el poder de fascinar a los demás. Ed. Debate.

KARL, M. (2006). Manuscritos económico-filosóficos de 1844. Buenos Aires. Ediciones Colihue S.R.L.

LAURIN-FRENETTE, N. (1976). Las teorías funcionalistas de las clases sociales. Sociología e ideología burguesa. Madrid. Siglo XXI de España Editores.

LÓPEZ-ARANGUREN, E. (2005). Problemas sociales: desigualdad, pobreza, exclusión social. Madrid. Editorial Biblioteca nueva.

MEIRIEU, P. (2001). La opción de educar. Ética y pedagogía. Octaedro.

MICHÉA, J. C. (2002). La escuela de la ignorancia. Madrid. Acuarela.

NEVEU, E. (2000). Sociología de los movimientos sociales. Quito-Ecuador. Editorial Abya Yala. 
OFICINA INTERNACIONAL DEL TRABAJO EN GINEBRA (2002). El trabajo decente y la economía informal. Conferencia Internacional del trabajo. $90^{a}$ reunión. Informe IV.

PUJOL I VILLALONGA, R. M. (1996). Educación y consumo. La formación del consumidor en la escuela. Barcelona. Editorial Horsori.

ROLLANO VILABOA, D. (2004). Cómo enfocar la educación hacia la ética. Vigo. Ideas Propias.

ROMÁN CUARTANGO, H. (2005). Filosofia y modernidad. España. Ediciones Intervención cultural.

VARIS, T. (2010). "Communicatios and new literacies in the multicultural world". En: Historia y Comunicación Social, № 15. Madrid: UCM. p.13-26.

VIDAL JIMÉNEZ, R. (2009). “UUna sociologogia (postmoderna) del miedo?” En: Historia y Comunicación Social, № 14, Madrid: UCM. p.315-316.

\section{Los autores}

M A Aurora López López, es doctora en Comunicación Audiovisual y Publicidad, y profesora de la Universidad de Valladolid en el Grado de Publicidad y Relaciones Públicas. A su vez compagina su labor docente con otra de carácter investigador, a través del grupo de investigación validado "Tendencias en Publicidad y Consumo" de la Asociación Científica ICONO 14-Universidad Complutense de Madrid. Entre sus materias de estudio destacan: la sociedad de consumo de masas, el sistema de distribución comercial, el marketing y la industria del lujo, la alimentación, la desigualdad social, la educación en valores, la antropología cultural y económica, entre otros. Por señalar algunas de sus publicaciones más recientes: La televisión y la construcción sociocultural en las sociedades democráticas (2012, UNPL, Argentina, Buenos Aires); Publicidad y Negocio. Retrospección introspectiva del negocio publicitario: el lujo objeto y el pecado capital señal (en RODRIGO MARTIN, L. (COORD.) (2012). Los maridajes de la publicidad. Las relaciones de la publicidad con otros ámbitos del conocimiento. Madrid. Editorial Icono14); Crisis y creatividad: economía en equilibrio. El ejemplo de los clusters (en Revista cientifica digital Creatividad y Sociedad. $\mathrm{N}^{\circ}$ 18. Junio de 2012. ISSN: 1887-7370. En www.creatividadysociedad. com.)

Isabel Rodrigo Martín: Licenciada en Publicidad y Relaciones Públicas por la Universidad de Valladolid en 2006. En esta misma universidad presentó su tesis doctoral "Arte, Comunicación y Propaganda. La vida y obra de Eduardo Vicente", obteniendo el Premio Extraordinario de Doctorado en 2012 y el título de doctora en Comunicación Audiovisual y Publicidad. En la actualidad es profesora del departamento de Historia Moderna, Contemporánea, de América. Periodismo. Comunicación Audiovisual y Publicidad. Participa en el proyecto de investigación "Comunicación, Diseño y Defensa" y obtuvo en premio de investigación Caja España 2007. Es miem- 
bro del Grupo de Investigación "Nuevas Tendencias en Publicidad y Consumo", de la Asociación Científica Icono 14. Su experiencia profesional se ha desarrollado en diversos medios de comunicación y como diseñadora gráfica. También ha asistido y participado en la organización de diferentes congresos y seminarios de las áreas de Comunicación y Diseño. Cuenta con publicaciones en estas mismas áreas en varias revistas y libros especializados.

Luis Rodrigo Martín. Doctor en Comunicación Audiovisual y Publicidad, y profesor de la Universidad de Valladolid en el Grado de Publicidad y Relaciones Públicas. Ha coordinado diferentes libros en el ámbito de la publicidad y la comunicación, así como diferentes congresos y simposios de carácter científico. Ha sido profesor invitado de prestigiosas universidades internacionales como la UNICAMP de São Paulo en Brasil, por destacar la última de sus colaboraciones. En la actualidad es asesor de la editorial del centro universitario Villanueva, a través de la cual ha conseguido publicar su primer libro de tipo científico y profesional en solitario: La sociedad de consumo en España (2013), con prólogo del prestigioso Catedrático en Publicidad el Dr. D. Raúl Eguizábal Maza de la Universidad Complutense de Madrid. 